\title{
REVISIONES
}

\section{Políticas de responsabilización por desempeño escolar: Un debate a partir de la literatura internacional y el caso chileno*}

\author{
School accountability policies: \\ A debate based on international literature and the Chilean situation \\ Responsabilidade política para o desempenho escolar: \\ Uma discussão da literatura internacional e o caso chileno \\ Alejandra Falabella, ${ }^{a}$ Luis Felipe de la Vega ${ }^{b}$ \\ a Centro de Investigación y Desarrollo en Educación (CIDE), Universidad Alberto Hurtado \\ Telf.: (56)228897103. Correo electrónico: afalabel@uahurtado.cl \\ ${ }^{\mathrm{b}}$ Candidato a Doctor en Educación, Pontificia Universidad Católica de Chile
}

\begin{abstract}
RESUMEN
Con la instalación del Sistema Nacional de Aseguramiento de la Calidad, Chile ha entrado en una etapa que consolida un modelo de responsabilización por desempeño. Este artículo ofrece un análisis conceptual de los supuestos y componentes en juego de este enfoque, así como de los principales resultados de investigaciones, tanto a nivel internacional como nacional. Para este fin se realizó una revisión de publicaciones en revistas científicas (Scopus, ISI) de los últimos 10 años y de libros y otros textos de autores clave; en total se consideraron 171 documentos. El artículo concluye que, a pesar de las altas expectativas de las políticas referidas, hay significativos nudos críticos que ponen en cuestión los beneficios esperados. Adicionalmente, se propone una diversificación de las concepciones sobre "rendición de cuentas", con el fin de ampliar los imaginarios políticos en educación y repensar el sistema de aseguramiento de la calidad en Chile.
\end{abstract}

Palabras clave: responsabilización por desempeño, rendición de cuentas, evaluación escolar, políticas de estandarización, Chile.

\begin{abstract}
Chile has entered into a stage that consolidates a model of high-stake accountablity through the setup of the National Quality Assurance System. This paper offers a conceptual analysis of the assumptions and components at play in the mentioned policy. In addition, the analysis of a relevant body of international and national research is presented as a review of publications in scientific journals (Scopus, ISI) for the past 10 years, plus books and other works by key authors. In total 171 documents were considered. The paper concludes that, despite the high expectations of accountability policies, there are critical issues that challenge the expected benefits. Additionally, the notion of "accountability" is opened and diversified, in order to rethink the Quality Assurance schema in Chile and, hence, expand the policy imaginary in education for future transformations.
\end{abstract}

Key words: high stakes accountability, testing, standardization, Chile.

Apoyo financiero: Fondecyt, proyecto $\mathrm{N}^{\circ} 11140302$, Gobierno de Chile. 
En las últimas décadas, las políticas de estandarización y responsabilización por desempeño (conocidas en inglés como accountability) han proliferado por distintos lugares del mundo, lo que ha transformado los modos de regular los sistemas educativos. La tendencia de estas reformas ha involucrado políticas tales como una mayor centralización del currículum, la definición de estándares de desempeño, sistemas de evaluación nacional de aprendizajes, información pública de resultados por escuela, así como consecuencias por dichos resultados (premios y sanciones).

Estas transformaciones emergen, con distintos grados de intensidad, junto a medidas que introducen principios de mercado y competencia en el servicio de la educación. De este modo, la libre elección de los padres y la introducción de financiamiento competitivo por alumno (voucher) usualmente se acopla a medidas de difusión de los resultados evaluativos por establecimiento. Asimismo, las políticas de privatización y de descentralización de la gestión escolar han ido de la mano con políticas que exigen una rendición de cuentas a los establecimientos por sus resultados académicos.

Estos cambios se dan, sin duda, con distintos matices, alcances y trayectorias históricas en cada contexto particular ${ }^{1}$. No obstante, es posible identificar patrones comunes en la nueva gramática de la política educativa, que se ha trasladado desde un modelo de bienestar centralizado y gestionado principalmente por el Estado, a un modelo de "cuasi-mercado" o "post-bienestar" (Gewirtz, 2002; Maroy, 2009). Ello implica una mixtura de políticas de centralización y de descentralización, en la que el Estado central delega la gestión escolar a servicios públicos y privados y, a su vez, crea nuevos modos de regulación y control por medio de la definición de metas, estándares y sistemas de evaluación nacional (Falabella, 2007, 2014a). El Estado, por tanto, no ha disminuido su poder bajo este esquema, sino que, más bien, ha cambiado el modo de ejercerlo; ha transitado desde un poder burocrático hacia un poder "a la distancia" (Rose y Miller, 1992). Esta situación se ha conceptualizado también como "gobernanza por resultados" (Newman, 2005) o "gobernanza vía números" (Ozga, 2011).

Este artículo analiza específicamente la política de responsabilización por desempeño en el escenario descrito. En el caso chileno, desde inicios de los años 80 se comenzaron a introducir estas medidas a través de la implementación de una evaluación estandarizada censal. En 1982 se instauró la Prueba de Evaluación del Rendimiento escolar (PER)², que derivó en 1988 en el Sistema de Medición de la Calidad Educativa (SIMCE). La política de responsabilización fue expandiéndose gradualmente y logró consolidarse, casi treinta años más tarde, con la Ley de Aseguramiento de la Calidad en 2011, que crea una nueva matriz institucional y una serie de dispositivos que conforman el actual modelo de rendición de cuentas por desempeño.

A pesar de la importancia que ha tenido esta política en el país, en Chile solo existe una incipiente discusión e investigación al respecto. Es por ello que el objetivo de este artículo es analizar conceptualmente la política de rendición de cuentas y presentar un meta-análisis de la literatura internacional y la referida al caso chileno, con el fin de contribuir al debate.

\footnotetext{
Por ejemplo, Estados Unidos ha creado fuertes políticas de evaluación y medidas de rendición de cuentas, mientras que las políticas de privatización y financiamiento competitivo han progresado de modo más lento (aunque creciente) y focalizado en ciertos estados. Mientras tanto, países como Inglaterra y Nueva Zelanda han ido acoplando de forma mucho más nítida esquemas de competencia, vía financiamiento por alumno, junto a políticas de estandarización y rendición por desempeño.

$2 \quad$ Entre 1968 y 1971 se aplicó la "Prueba Nacional", destinada a medir los logros de aprendizaje de los estudiantes en 8vo básico, pero debido a la falta de capacidad técnica de la época, se descontinuó.
} 
La metodología empleada implicó un estudio de publicaciones científicas de los últimos 10 años (2004-2014) indexadas en Scopus o Web of Science (ISI). Como criterio de inclusión de los textos se consideró la presencia de análisis o evaluación — teórica y/o empírica - respecto de políticas de responsabilización escolar como aspecto central de estudio del documento ${ }^{3}$. Los criterios de exclusión correspondieron a la incorporación secundaria de estas políticas en el análisis (por ejemplo, trabajos referidos a reformas educativas en general) y los trabajos referidos a la rendición de cuentas, pero en otras áreas de política pública. Se consideraron, además, textos y libros de académicos reconocidos en el área que han abordado el tema. Se revisaron 110 artículos con resultados empíricos, 28 ensayos, 3 meta-análisis de la literatura, 21 libros, 6 informes de estudio y 2 tesis doctorales, es decir, un total de 171 documentos.

El presente artículo consta de cinco secciones. En la primera se describen tres enfoques desde los cuales es posible abordar la responsabilización escolar. En la segunda se analiza en detalle el enfoque de responsabilización por desempeño o rendición de cuentas con altas consecuencias, ya que es el más cercano a lo que se implementa en Chile. En la tercera se examinan los efectos y nudos críticos vinculados a esta política, en base a la revisión de la literatura sobre el tema. En la cuarta se describen las características del caso chileno y se examinan los estudios que existen al respecto. Finalmente, se realizan conclusiones referidas al caso chileno.

\section{ENFOQUES DE POLÍTICAS DE RESPONSABILIZACIÓN EDUCATIVA}

A pesar de que las medidas de responsabilización por desempeño o "con altas consecuencias" son una tendencia creciente en los sistemas escolares, esto no significa que sean el único modo de rendición de cuentas. Es por esto que, para comprender y debatir respecto a las políticas de responsabilización escolar, es necesario, en primer lugar, comprender que existen distintos enfoques al respecto.

La responsabilización —o rendición de cuentas - hace referencia a que las escuelas son responsables de la oferta que proveen. Dependiendo del modelo educativo de cada país, este mecanismo se ha configurado de distintos modos, que dan cuenta de aspectos cruciales como: ¿quién asume las responsabilidades?, ¿qué responsabilidades se asumen?, ¿a quién se le rinde cuenta por dicha responsabilidad?, ¿cómo el Estado asegura a la población una educación confiable y de calidad?

Estas preguntas permiten identificar al menos tres enfoques distintos ${ }^{4}$. Primero, el denominado responsabilización estatal (también conocido como responsabilización burocrática), ligado al Estado de bienestar, predominante entre los años 40 y 80, y presente actualmente en países como Francia, Italia y Portugal. Segundo, el enfoque de responsabilización por desempeño, que comenzó gradualmente desde los años 80 y que se ha desarrollado en países como Chile, Colombia, Inglaterra, Estados Unidos y Nueva Zelanda. Tercero, el enfoque de responsabilización profesional, que involucra importantes

Las palabras de búsqueda utilizadas fueron: accountability, rendición de cuentas, responsabilización escolar, evaluación escolar, estandarización.

4 Para profundizar en estos enfoques ver también Carrasco, Falabella y Seppänen (2015), Darling-Hammond (1994), Hargreaves y Shirley (2012). 
regulaciones estatales relacionadas a la formación docente y sus condiciones laborales y una gestión local fortalecida. Se identifica en países nórdicos como Finlandia y también, aunque en menor medida, en Canadá, Escocia y Bélgica. A continuación se presenta una breve descripción de cada enfoque, la que luego se sintetiza en la Tabla 1.

Responsabilización estatal. En este enfoque el Estado central es responsable de la oferta educativa, la que se orienta por el principio de igualdad y por el derecho de servicios sociales universales. La escuela, enmarcada en un esquema centralizado y jerárquico, debe rendir cuentas a los niveles de administración superior por la provisión del derecho a la educación, la implementación de un currículum nacional y la aplicación de normativas. El foco está puesto, por tanto, en el control de las condiciones de entrada del sistema (inputs), no en los resultados (outputs). Lo anterior implica que las escuelas son parte de una estrategia educativa más amplia, que se corresponde con un proyecto de educación pública.

En este contexto, la figura del docente cumple un rol de gran relevancia para las familias y para la ciudadanía en general; se le otorga confianza por su experticia y autoridad. Generalmente, el involucramiento y participación de los padres en las decisiones escolares es limitada.

Responsabilización por desempeño escolar. Bajo este enfoque, los establecimientos educativos suelen tener mayor grado de autonomía en su gestión financiera y administrativa, pero, ello se da a cambio de rendir cuentas al Estado por su desempeño en las evaluaciones nacionales. En concreto, esta política involucra una combinación de estrategias, fases y dispositivos que incluye: i. metas de resultados prefijadas desde el Estado; ii. sistemas de evaluación estandarizada de la oferta de la calidad y/o de los resultados de aprendizaje; iii. publicación de resultados y sistema de clasificación de la calidad de las escuelas; iv. consecuencias por resultados (premios y sanciones); usualmente, v. recursos y/o asesoría técnica para aquellos establecimientos que obtienen un rendimiento insatisfactorio. Este enfoque es conocido en la literatura estadounidense como high stakes accountability y en la inglesa como performative accountability.

Como se ha mencionado, lo anterior suele sumarse a la exigencia de que la escuela rinda cuentas por sus resultados también a las familias, en el marco de un sistema orientado hacia el mercado. Se espera, de este modo, que los padres sean reguladores de la calidad del sistema educativo en base a información "transparente" y así puedan elegir, demandar y, eventualmente, desertar de una escuela ("el poder del cliente").

Responsabilización profesional. Este enfoque apunta a que el equipo escolar se responsabilice por brindar experiencias de aprendizaje de calidad, lo que depende del desarrollo sostenible de las capacidades profesionales dentro de la escuela. La rendición de cuentas se orienta hacia las comunidades educativas — accountability interno-y al Estado local y/o nacional, es decir, responsabilización pública. Pueden existir sistemas externos de evaluación y de rendición, pero estos tienden a ser flexibles, no están asociados a políticas de competencia entre escuelas ni a bonos individuales o sanciones y los resultados no son publicados en los medios.

Los esfuerzos de este tipo de política se orientan a la obtención de altos estándares en la formación docente y en sus barreras de entrada, junto a un alto status social de la profesión y condiciones laborales atractivas. Esto se combina con un bajo nivel de estructuración del currículum nacional, la existencia de gobiernos locales fuertes que otorgan apoyo estable y una confianza de base en las capacidades institucionales. Este modelo se ha desarrollado en países con altos niveles de equidad social y acuerdos nacionales respecto al valor por la educación y el trabajo docente. 
Tabla 1. Enfoques de responsabilización educativa

\begin{tabular}{|c|c|c|c|}
\hline & \multicolumn{3}{|c|}{ Enfoque } \\
\hline Dimensión & $\begin{array}{c}\text { Responsabilización } \\
\text { Estatal }\end{array}$ & $\begin{array}{l}\text { Responsabilización } \\
\text { por desempeño escolar }\end{array}$ & $\begin{array}{l}\text { Responsabilización } \\
\text { profesional }\end{array}$ \\
\hline $\begin{array}{l}\text { Modelo de } \\
\text { educación }\end{array}$ & $\begin{array}{l}\text { Estado de bienestar, mo- } \\
\text { delo centralizado. Aso- } \\
\text { ciado a países como } \\
\text { Francia, Italia, Portugal. }\end{array}$ & $\begin{array}{l}\text { Cuasi-mercado, Estado } \\
\text { subsidiario. Asociado a } \\
\text { reformas en EE.UU., In- } \\
\text { glaterra, Chile, Colom- } \\
\text { bia. }\end{array}$ & $\begin{array}{l}\text { Educación pública forta- } \\
\text { lecida, flexibilidad curri- } \\
\text { cular y espacio de auto- } \\
\text { nomía local. Puede con- } \\
\text { tar con provisión mixta y } \\
\text { libre elección regulada. } \\
\text { Asociado a países nórdi- } \\
\text { cos, Escocia y Bélgica. }\end{array}$ \\
\hline $\begin{array}{l}\text { Escuela es } \\
\text { responsable } \\
\text { de: }\end{array}$ & $\begin{array}{l}\text { Derechos sociales, aten- } \\
\text { der cobertura, implemen- } \\
\text { tar currículum nacional, } \\
\text { responder a la burocracia } \\
\text { estatal. }\end{array}$ & $\begin{array}{l}\text { Desempeño en pruebas } \\
\text { estandarizadas e inspec- } \\
\text { ciones externas, satisfac- } \\
\text { ción demanda de fami- } \\
\text { lias. }\end{array}$ & $\begin{array}{l}\text { Calidad de los procesos } \\
\text { educativos, desarrollo de } \\
\text { las comunidades profe- } \\
\text { sionales (juicio profesio- } \\
\text { nal y criterio contexto). }\end{array}$ \\
\hline $\begin{array}{l}\text { Mecanismos de } \\
\text { regulación }\end{array}$ & $\begin{array}{l}\text { Sistema de supervisión y } \\
\text { control sobre estableci- } \\
\text { mientos mayoritariamen- } \\
\text { te públicos. }\end{array}$ & $\begin{array}{l}\text { Sistema de rendición de } \\
\text { cuentas al Estado por re- } \\
\text { sultados evaluativos. } \\
\text { Competencia entre diver- } \\
\text { sidad de proveedores. }\end{array}$ & $\begin{array}{l}\text { Altas barreras de entrada } \\
\text { para la profesión docente } \\
\text { y proveedores de la edu- } \\
\text { cación. Sistemas perma- } \\
\text { nentes de acompaña- } \\
\text { miento a las escuelas. }\end{array}$ \\
\hline $\begin{array}{l}\text { Modelo de } \\
\text { evaluación }\end{array}$ & $\begin{array}{l}\text { Evaluación centrada en } \\
\text { cumplimiento de indica- } \\
\text { dores y procedimientos } \\
\text { formales. Evaluaciones } \\
\text { externas (muestrales o } \\
\text { censales) no son publica- } \\
\text { das. }\end{array}$ & $\begin{array}{l}\text { Evaluación estandariza- } \\
\text { da, censal, publicación } \\
\text { de resultados por escuela. }\end{array}$ & $\begin{array}{l}\text { Evaluación interna y lo- } \\
\text { cal, participación de las } \\
\text { comunidades educativas. } \\
\text { Evaluaciones externas } \\
\text { (muestrales o censales) } \\
\text { no son publicadas. }\end{array}$ \\
\hline $\begin{array}{l}\text { Escuela le } \\
\text { rinde cuenta a: }\end{array}$ & $\begin{array}{l}\text { Estado central. El docen- } \\
\text { te es una figura de autori- } \\
\text { dad y experto; baja rendi- } \\
\text { ción a los padres. }\end{array}$ & $\begin{array}{l}\text { Estado y familias por re- } \\
\text { sultados evaluativos }\end{array}$ & $\begin{array}{l}\text { Comunidades educativas } \\
\text { "accountability interno", } \\
\text { y al Estado local y/o na- } \\
\text { cional, "responsabiliza- } \\
\text { ción pública". }\end{array}$ \\
\hline Consecuencias & $\begin{array}{l}\text { Sanciones dentro de una } \\
\text { jerarquía institucional. }\end{array}$ & $\begin{array}{l}\text { Premios y sanciones por } \\
\text { desempeño escolar; pue- } \\
\text { den ser individuales e } \\
\text { institucionales, simbóli- } \\
\text { cos y/o materiales }\end{array}$ & $\begin{array}{l}\text { Intervención o decisiones } \\
\text { de cambio de personal } \\
\text { desde el gobierno local; } \\
\text { puede quitársele licencia } \\
\text { a sostenedores privados }\end{array}$ \\
\hline
\end{tabular}


Es importante notar que los sistemas educativos de cada país combinan componentes de los distintos enfoques analizados; sin embargo, generalmente predomina un modelo sobre los otros. En el caso chileno se ha incrementado la opción por la segunda alternativa —responsabilización por desempeño-; es por ello que se profundiza en este enfoque en los siguientes apartados.

\section{COMPONENTES DE LA POLÍTICA DE RESPONSABILIZACIÓN POR DESEMPEÑO ESCOLAR}

Este enfoque implica un conjunto de medidas enlazadas que esperan movilizar en los actores acciones para el logro de determinados estándares y resultados preestablecidos. A la política subyace un conjunto de componentes y supuestos para el logro de su efectiva implementación. A continuación estos se analizan en mayor detalle.

Calidad: medible y comparable. Evaluar la calidad de cada establecimiento es un eje clave de modelo para poder establecer la rendición de cuentas de las escuelas y motivar el mejoramiento continuo. Es importante notar que desde esta perspectiva se asume que la calidad educativa puede ser consensuada y estandarizada, y que el grado de logro puede ser medido. Asimismo, que es posible y necesario comparar y jerarquizar estos niveles de logro de la calidad en base a estándares nacionales. El énfasis, generalmente, está puesto en medir los aprendizajes de los alumnos como indicador de calidad, aunque a veces estas políticas también incluyen evaluaciones de la oferta escolar.

Equidad: estándares nacionales, "No hay excusas". Los actores escolares son entendidos como responsables de los resultados evaluativos de los alumnos, en consecuencia, se particulariza la responsabilidad a nivel institucional. Generalmente, esta perspectiva solo considera de forma marginal o secundaria las condiciones estructurales y contextuales de las escuelas, el origen socio-cultural de los alumnos, los recursos para la contratación de docentes, así como la responsabilidad que le caben tanto al Estado local como al central. La calidad de la oferta se define, entonces, de manera independiente del contexto específico, aunque pueden existir mecanismos más o menos sofisticados en cada país para controlar variables como el nivel socio-económico de los alumnos y el "efecto par".

En este sentido, este enfoque asume una noción de equidad en la que todos los establecimientos son responsables por igual de sus resultados y deben rendir cuentas por ello. Estas políticas han ido de la mano de discursos que han promovido partes del ideario de la teoría de las escuelas eficaces, planteando que "no hay excusas" para escuelas con bajo rendimiento académico y que las escuelas pueden "hacer una diferencia", independiente de la composición social y étnico/racial de los alumnos ${ }^{5}$.

Diferenciación, clasificación y competencia como motor de mejora. La evaluación estandarizada establece y muestra públicamente diferencias entre establecimientos; así se permite la elección informada de los padres y la competencia entre establecimientos por mejores resultados académicos. En otras palabras, para estimular procesos de mejoramiento

La promoción de estas ideas nacen como respuesta crítica al llamado "pesimismo sociológico" de los años 60, en que se planteó la "teoría de la reproducción social" de Bourdieu y Passeron (1990, 1era edición 1970) y se publicó el Informe Coleman (1966). Ambos textos, uno de corte teórico y el otro empírico, argumentan una significativa incidencia de los componentes estructurales de la sociedad en el funcionamiento de una escuela y en los resultados de aprendizaje de los alumnos. 
educativo, el diseño de la política requiere crear mecanismos de diferenciación $n^{6}$, clasificación y ordenamiento. En contraposición, existen modelos que sitúan el foco en definir requisitos mínimos y obligatorios para todos los establecimientos y, por ende, no se establecen grados diferenciados de logro, sino que se intenta lograr condiciones de igualdad bajo un marco de políticas universales ${ }^{7}$.

Gestión racional. Este modelo promueve la idea de una gestión orientada a logros, en que se fijan metas respecto de los resultados en pruebas estandarizadas y otros indicadores establecidos, se realizan monitoreos sistemáticos y se toman decisiones fundamentadas en "evidencias" derivadas de los resultados evaluativos ("data-driven decision-making"). Desde esta perspectiva se le otorga una alta confianza y legitimidad a los instrumentos de evaluación estandarizada y sus resultados, los que señalan a las instituciones cómo deben orientar la gestión de acuerdo a los aspectos menos logrados. Por tanto, los resultados evaluativos son decisivos para determinar las prioridades de la gestión pedagógica. Se realiza, usualmente, escasa referencia a la necesidad de una reflexión curricular docente sobre qué se evalúa externamente y cómo ello se engarza o no al proyecto educativo local y sus principios pedagógicos y/o filosóficos.

Motivación extrínseca. Los resultados por escuela, docente y/o alumno están ligados a un conjunto de incentivos y sanciones (de orden económico, normativo y simbólico), con los que se busca estimular el logro de estándares nacionales y el incremento en resultados de pruebas estandarizadas. Se asume que los individuos se motivan y movilizan positivamente por medio de des/incentivos externos y mecanismos de competencia (por ejemplo, ranking, "ordenamiento" de la calidad escolar, bonos individuales o colectivos, etc.), lo que acerca las acciones individuales a las expectativas del sistema. Normalmente, estas políticas hacen escasa referencia a compromisos internos, vocación, solidaridad entre escuelas u otros conceptos similares.

El conjunto de los supuestos analizados anteriormente refleja una visión "racional" de la gestión escolar, en la que las metas y evaluaciones estandarizadas componen un eje rector y existe una expectativa causal entre los elementos de la política que conllevarían a un "circuito virtuoso de mejora continuo".

\section{NUDOS CRÍTICOS DE LA POLÍTICA DE RESPONSABILIZACIÓN POR DESEMPEÑO ESCOLAR}

\subsection{REVISIÓN DE LA LITERATURA}

Dado el marco anterior surge la pregunta: ¿en qué medida la política de responsabilización con altas consecuencias gatilla los cambios esperados? Particularmente interesa preguntar por los beneficios, efectos y tensiones que ha implicado la política y si cumple con las expectativas planteadas. En base a una extensa revisión bibliográfica, interesa identificar nudos críticos en los que se organiza el debate en esta materia. A continuación, se abordarán cuatro puntos respecto de los efectos de la política que refieren a calidad educativa, equidad, gestión escolar y profesión docente.

Para una mayor discusión, ver Lazzarato (2012).

Tales como distribución centralizada de los docentes, iguales sueldos y condiciones laborales. 
Prácticas pedagógicas: ¿Mejora la calidad? La pregunta relativa a si las políticas de responsabilización han generado mayor calidad y efecto positivo en el aprendizaje es un primer nudo crítico, sin respuesta única. Autores que defienden los beneficios de este modelo concuerdan en que contribuye a garantizar que los alumnos reciban una educación de calidad (ver, por ejemplo, Hanushek y Woessmann, 2010; Mourshed, Chijioke y Barber, 2010; Puryear, 2006). La principal virtud de este enfoque sería su orientación al logro.

Desde esta perspectiva se comprende que la acción conjunta de los componentes de la política debiera propender hacia el aseguramiento de la calidad; Manno (2005) llama a esto "cadena de valor del desempeño", la que motivaría a ajustar y corregir acciones y aproximarlas a la expectativa que existe sobre lo que deben lograr las escuelas (p. 3). Asimismo, di Gropello (2004) y Winkler (2006) argumentan que el potencial de esta estrategia está en que genera un accountability doble, pues las instituciones escolares deben rendir cuenta no solamente al aparato estatal, sino también a las familias, lo que refuerza los mecanismos de aseguramiento de la calidad. Se valora, asimismo, la oportunidad de favorecer la autonomía escolar con el fin de elaborar estrategias locales para alcanzar los objetivos esperados.

Algunos estudios respecto a los aprendizajes de los alumnos han encontrado resultados positivos, aunque leves. Por ejemplo, Carnoy y Loeb (2003) y Yaekyoung (2008), en referencia al caso estadounidense, Barber (2004) para al caso de Estados Unidos e Inglaterra, y un conjunto de estudios realizados por el Banco Mundial, que incluye India, Pakistán, Uganda y Liberia (Bruns, Filmer y Patrinos, 2011). Sin embargo, estos mismos autores han tratado el tema con cautela. Incluso el propio Barber, quien asesoró el diseño de este tipo de medidas bajo el gobierno de Tony Blair, matizó posteriormente sus conclusiones al incorporar la idea de que no todos los sistemas requieren de la misma intensidad en términos de presión (Barber, 2010).

Estudios desarrollados por Figlio y Loeb (2011) y el National Research Council (2011), a partir de revisiones de investigaciones relacionadas con la implementación del accountability educacional en Estados Unidos, no lograron identificar una mejora luego de más de 10 años de la instalación de este tipo de políticas. A un resultado similar llegan Dee et al. (2010), quienes no identifican aumentos estadísticamente significativos en los puntajes de los estudiantes en los test estandarizados, luego de 9 años de implementación de la Ley No Child Left Behind. Asimismo, Dupriéz y Dumay (2011), utilizando datos comparativos entre países de la prueba PISA, indican que no hay evidencia para sostener que la política de accountability genere mayores resultados académicos. De hecho, países como Canadá, Finlandia, Singapur y Japón se destacan por obtener altos grados de rendimiento académico sin haber implementado las políticas en cuestión, en contraposición con países como Inglaterra y Estados Unidos.

Por otra parte, estudios cualitativos y mixtos han entregado evidencias sobre el efecto que ha tenido este tipo de políticas en las prácticas internas en las escuelas. En el caso de Estados Unidos, se ha encontrado que promueven positivamente que los equipos escolares recojan y utilicen evidencias tanto externas como internas para sus procesos de planificación y mejora (Hamilton et al., 2007). Asimismo, se identifica que los docentes realizan mayor seguimiento y monitoreo de sus alumnos.

No obstante, estos mismos estudios, junto a una amplia literatura tanto de Inglaterra como de Estados Unidos, dan cuenta de la existencia de efectos negativos en las prácticas pedagógicas y en la calidad del proceso educativo. Por ejemplo, se observan prácticas como: ajustes y reducción curricular de acuerdo a las materias evaluadas; adiestramiento a los alumnos en las pruebas estandarizadas (conocido como "teaching to the test"); aumento 
de tiempo y recursos en las asignaturas y niveles evaluados; tácticas tramposas que faltan a la ética (por ejemplo, excluir de los tests alumnos con bajo rendimiento o calificarlos como niños con necesidades educativas especiales). Entre los estudios que han evidenciado estos efectos se encuentran los de Anderson (2011); Dee et al. (2010); Ehren y Hatch (2015); Firestone, Schorr y Monfils (2004); Mintrop y Trujillo (2007)

Como consecuencia de lo anterior, se ha detectado que existe una "inflación de puntaje" en pruebas estandarizadas (Jacob, 2009; Koretz, 2005), es decir, alzas en los resultados que no se relacionan necesariamente con mejoras de aprendizaje en las asignaturas. Es por esto que los autores señalan que se ha de interpretar con precaución las alzas de resultados, pues este fenómeno las distorsiona y reduce su validez.

Equidad e inclusión social. Aunque las políticas de rendición de cuentas han promovido la expectativa de que se logrará mayor equidad en los logros de todos los alumnos, este punto ha sido otro nudo controvertido en la literatura especializada. Autores como Ball (2006), Gewirtz (2002) y Power y Frandji (2010) han argumentado que las políticas de accountability, siguiendo una lógica de competencia, incentivan y premian la selección de aquellos alumnos con mayor rendimiento académico y, a la vez, castigan a las escuelas que reciben a los alumnos de menor rendimiento y de sectores de mayor pobreza y marginalidad. Estudios como los de Dupriez y Dumay (2011) confirman que las reformas que han motivado la privatización, las políticas de libre elección parental y la publicación de resultados individuales por establecimiento han aumentado los grados de segregación social y educativa.

Se ha encontrado también que dentro de las escuelas se realizan prácticas de segregación interna que distribuyen los esfuerzos educativos de manera desigual como estrategia para aumentar los resultados en pruebas estandarizadas. Por ejemplo, se da menor atención a aquellos niños que no son evaluados por las pruebas nacionales o que están muy lejos de lograr los estándares exigidos. A su vez, los docentes focalizan sus esfuerzos en enseñarle a los alumnos "frontera", es decir, a aquellos que están más cerca de lograr el estándar exigido (Youdell, 2004). De este modo, se argumenta que el accountability relacionado con altas consecuencias genera una disminución de oportunidades de aprendizaje para algunos tipos de estudiantes, especialmente los que tienen menor logro- en su mayoría minorías étnicas o aquellos que viven en condiciones de pobreza - lo que finalmente redunda en la inequidad del sistema (Darling-Hammond, 2007, 2010).

Gestión escolar: ¿desarrollo interno o dar respuesta a exigencias externas? Existe una amplia discusión respecto a en qué medida el mejoramiento educativo se desarrolla "desde dentro" o "desde fuera" de la escuela, lo que pone en cuestión el nivel de prescripción y control externo de las políticas de estandarización. Autores como Hargreaves y Fink (2006) han argumentado que el cambio sostenible en educación requiere de ambos puntos de partida. Sin embargo, existe una visión crítica sobre las políticas en cuestión, pues en ellas predomina un alto nivel de estructuración externa, lo que deja un limitado espacio para el desarrollo interno de las escuelas. Hargreaves y Shirley (2012), por ejemplo, sostienen que en países como Estados Unidos e Inglaterra existe un desbalance, con un claro énfasis en la presión, en desmedro de un apoyo cercano y efectivo a los equipos escolares.

\footnotetext{
En este último estudio se examinaron las prácticas desarrolladas en nueve escuelas de California con resultados excepcionalmente altos o bajos. Interesaba contrastar los factores internos en la gestión escolar y los procesos de mejora de estos dos tipos de escuela. Para sorpresa de los investigadores, las prácticas de ambos tipos de escuela eran más bien similares entre sí, por lo que los resultados son más bien dispersos. Sin embargo, lo que sí se asocia a escuelas con altos resultados es la alineación entre el currículum y las prácticas de aula con los contenidos y habilidades evaluadas en las pruebas estatales.
} 
Desde una perspectiva similar, MacBeath (2011) plantea que la estandarización de las políticas de reponsabilización por desempeño introduce una visión externa a la escuela en cuanto a las pautas y formas de plantear procesos de diagnóstico, planificación y análisis de las propias prácticas; predomina, así, el "locus externo". Según el autor, esta situación rigidiza los procesos internos de las instituciones y dificulta contextualizar el trabajo escolar y responder a las necesidades locales. Además, genera resistencias desde los docentes y aminora el desarrollo de capacidades institucionales?

Rol de los docentes: ¿desarrollo profesional o tecnificación? Otro punto relevante respecto de las reformas en cuestión refiere a la manera en que estas impactan en el trabajo docente. Darling-Hammond (2010), Darling-Hammond, Wilhoit y Pittenger (2014) señalan que el principal factor de mejoramiento de todo el sistema educativo es contar con docentes bien preparados y que estén concentrados en mejorar sus propias prácticas. Sin embargo, la autora argumenta que las políticas de evaluación estandarizada y de accountability priorizan el control y la amenaza por sobre el profesionalismo, la vocación y la responsabilidad moral. Esto refleja una desconfianza profunda hacia quienes trabajan en las escuelas y asume que ellos trabajarán de modo satisfactorio solo si existe un potencial premio o castigo.

Además, esta situación deriva en una desprofesionalización de los docentes, quienes se vuelven actores ejecutores más que profesionales de la enseñanza. Se ha generado también una creciente burocratización del quehacer docente, lo que ha minimizado los espacios de reflexión e innovación. En la misma línea, un conjunto de estudios evidencia que estas políticas han afectado negativamente la autoestima profesional de los docentes, la motivación por el trabajo y los niveles de estrés (Finnigan y Gross, 2007; MacDermott, 2007; Valli y Buese, 2006; Woods y Jeffrey, 2002).

En base a la revisión de la literatura, el análisis de las políticas en cuestión y los debates que existen al respecto, es posible, entonces, identificar cuatro tensiones, las que se sintetizan en la Tabla 2.

Tabla 2. Tensiones que emergen en la escuela a propósito de la política de responsabilización por desempeño

\begin{tabular}{|c|c|c|}
\hline Dimensión & \multicolumn{2}{|c|}{ Tensiones entre } \\
\hline $\begin{array}{l}\text { Calidad de } \\
\text { las prácticas } \\
\text { pedagógicas }\end{array}$ & $\begin{array}{l}\text { Énfasis en los procesos y formación } \\
\text { integral. Innovación pedagógica y } \\
\text { contextualización de las prácticas. }\end{array}$ & $\begin{array}{l}\text { Estandarización de las prácticas de enseñan- } \\
\text { za y evaluación. Currículum orientado y re- } \\
\text { ducido al logro de resultados medibles. }\end{array}$ \\
\hline $\begin{array}{l}\text { Equidad e } \\
\text { inclusión }\end{array}$ & $\begin{array}{l}\text { Educación inclusiva como enfoque } \\
\text { de enseñanza-aprendizaje. }\end{array}$ & $\begin{array}{l}\text { Estrategias de selección/expulsión, segrega- } \\
\text { ción intra-escuela según niveles de logro. }\end{array}$ \\
\hline $\begin{array}{l}\text { Gestión } \\
\text { Escolar }\end{array}$ & $\begin{array}{l}\text { Desarrollo de capacidades internas } \\
\text { y trabajo colaborativo. Énfasis en la } \\
\text { autoevaluación; prima "locus inter- } \\
\text { no". }\end{array}$ & $\begin{array}{l}\text { Aumento en los mecanismos de evaluación } \\
\text { y control interno. Énfasis en los cambios de } \\
\text { corto plazo, que generen resultados medi- } \\
\text { bles y observables. Prima "locus externo". }\end{array}$ \\
\hline $\begin{array}{l}\text { Desarrollo } \\
\text { Profesional } \\
\text { Docente }\end{array}$ & $\begin{array}{l}\text { Profesionalización docente, toma } \\
\text { de decisiones fundadas en el con- } \\
\text { texto y criterio profesional. }\end{array}$ & Des-profesionalización, tecnificación. \\
\hline
\end{tabular}

El autor propone situar el foco de la gestión escolar en el locus interno, que refiere a una práctica permanente — transversal y longitudinal — de revisión de la propia acción, que involucre a la comunidad educativa. 


\subsection{DEBATES EN LA LITERATURA Y PERSPECTIVAS DE ANÁLISIS EN CONFRONTACIÓN}

En base a lo expuesto anteriormente, es importante notar que existen diferentes interpretaciones de los resultados que dan cuenta de al menos tres ángulos teóricos de análisis ${ }^{10}$. Las visiones más afines a las políticas de responsabilización por desempeño reconocen que ellas pueden generar "efectos no deseados" (Altrichter y Kemethofer 2015; di Gropello, 2004; Hamilton, Stecher y Yuan, 2012; Looney, 2009); sin embargo, consideran que ello es corregible y que el beneficio de la política es, en comparación, mayor. Recomiendan, por tanto, tomar resguardos que aseguren la adecuada inserción de la rendición de cuentas en el sistema educativo. Por ejemplo, sugieren cuidar la cantidad de incentivos y que existan múltiples instrumentos de evaluación (cuantitativos y cualitativos, evaluaciones en distintas asignaturas y niveles escolares, etc.), ya que reduce la posibilidad de prepararlos.

Por otra parte, intelectuales vinculados a la teoría de mejora escolar, como DarlingHammond (2010), Darling-Hammond et al., (2014), Hargreaves y Shirley (2012), MacBeath (2009) y Ravitch (2010), contra-argumentan lo dicho anteriormente, planteando que estos efectos no son producto de la implementación de componentes específicos, sino que responden a un diseño global del modelo. Concuerdan respecto a la importancia de evaluaciones externas que retroalimenten el trabajo educativo, pero acusan un desbalance en el diseño de este tipo de políticas, ya que ha exacerbado la prescripción y la sanción por sobre el desarrollo de habilidades internas de los equipos profesionales.

Finalmente, desde una perspectiva socio-crítica, los autores enfatizan que las políticas que se han analizado sitúan a las escuelas en una matriz competitiva, lo que ha transformado la subjetividad de los actores y los fines de la educación (Apple, 2003; Ball, 2006; Gewirtz, 2002; Lipman, 2004; Power y Frandji, 2010; Thrupp y Lupton, 2006). Desde esta perspectiva, se plantea que la preocupación por lograr las metas establecidas y demostrar a otros un desempeño "exitoso" ha colonizado la gestión escolar y el actuar diario de los docentes, pese a que existen resistencias y críticas de parte de los propios actores escolares. Como consecuencia, estos se encuentran en un lugar de tensión, entre atender las necesidades locales para asegurar aprendizajes profundos, por una parte, y lograr metas "visibles" que contribuyan al prestigio institucional en un escenario de cuasi-mercado, por otra.

\section{CONTEXTO NACIONAL: SISTEMA DE ASEGURAMIENTO DE LA CALIDAD}

El Sistema de Aseguramiento de la Calidad en Chile implica un entramado de políticas vinculantes que se han ido produciendo y entrelazando progresivamente desde la reforma educativa de los años 80. En esa época, en el marco de una transformación global hacia la constitución de un Estado subsidiario, se comienza a idear el diseño de un mercado escolar que combina elementos de competencia vía subvención por niño, libre elección escolar y una prueba nacional estandarizada que pudiese medir la calidad de cada escuela con el fin de orientar las decisiones de los padres, como también de la gestión escolar. Ejemplo de esto son los escritos del asesor del Ministerio de Hacienda de la época, Gerardo Jofré (1988), y del Ministro de Educación, Alfredo Prieto (1983).

10 Para un análisis en mayor detalle, ver Falabella (2014a). La autora distingue tres enfoques de investigación: "proaccountability", "mejora escolar" y "sociología post-critica". 
En la década del 90 se fortalecen los mecanismos orientados a informar la elección escolar de los padres, entre los que se destacan la publicación de los resultados del SIMCE en 1995, su publicación en el sitio web institucional en 2001, la entrega de cartillas para padres en 2005 y mapas online con información por escuela en 2010. También se crean, en 1996, incentivos salariales colectivos por indicadores de calidad (entre los que se incluye los resultados SIMCE), llamado Sistema Nacional de Evaluación de Desempeño. Paulatinamente, desde los años 2000 se comienza a dar mayor relevancia al incremento de resultados en las evaluaciones estandarizadas. En este marco, se incorpora el año 2000 el programa de Escuelas críticas y el 2006 el de Liceos prioritarios, los que supusieron la categorización de escuelas y la implementación de asesorías técnicas desde el sector privado como una política de afirmación positiva en establecimientos con bajos resultados y en contextos de alta vulnerabilidad social.

Posteriormente, con la Ley de Subvención Escolar Preferencial (SEP) en 2008, se implementan dispositivos que se orientan más explícitamente hacia un sistema de responsabilización por resultados y altas consecuencias para las escuelas. Con esta política se incorpora un subsidio diferenciado por estudiante, lo que compensa las desigualdades sociales de origen de los estudiantes denominados prioritarios. La entrega de estos recursos se asocia directamente con la exigencia de mayor calidad de servicio y el incremento de resultados académicos evaluados a través de indicadores medibles y estandarizados, lo que implica posibles sanciones por no cumplimiento de meta (por ejemplo, el eventual cierre de un establecimiento por rendimiento insatisfactorio reiterado). También es relevante mencionar que se extiende gradualmente el número de aplicaciones de la prueba SIMCE hacia diferentes niveles $\left(2^{\circ}, 4^{\circ}, 6^{\circ}, 8^{\circ}\right.$ básico, $2^{\circ}$ y $3^{\circ}$ medio) y asignaturas (Lenguaje y Comunicación, Matemática, Ciencias Naturales, Historia, Geografía y Ciencias Sociales, Educación Física, Inglés, TICs).

Finalmente, la Ley del Sistema de Aseguramiento de la Calidad (2011) inicia la instalación de una nueva institucionalidad, con la Superintendencia y Agencia de la Calidad, y un sistema de planificación, asesoramiento técnico, evaluación, ordenamiento e inspección para todos los establecimientos subvencionados del país. A ello se suman mecanismos de rendición de cuentas de los establecimientos hacia las familias y a las instituciones del Estado, junto con consecuencias jurídicas que se derivan de la aplicación de los instrumentos que provee la ley.

\subsection{ESTADO DE LA CUESTIÓN EN CHILE}

El debate sobre este tipo de políticas también se ha dado en Chile, aunque aún existe escasa evidencia sobre sus efectos. Uno de los escritos iniciales en la materia es el trabajo de Eyzaguirre y Fontaine (2008), que estudia un conjunto de escuelas de bajo rendimiento localizadas en contextos de pobreza. Su objetivo es analizar las prácticas de estos establecimientos educacionales que podrían explicar sus bajos puntajes. Con relación a los desafíos que se presentan para mejorar la experiencia educativa de estos alumnos, Eyzaguirre y Fontaine recomiendan justamente aumentar las medidas de "presión y apoyo"; en sus palabras: "probablemente un conjunto de medidas de presión y apoyo podría ayudarlas a afirmar su gestión, a mantener en el tiempo las buenas prácticas y a enfrentar las resistencias que normalmente se generan al instalar sistemas de gestión eficaces" (p. 312). 
Por su parte, el capítulo de Espínola y Claro (2010) destaca los beneficios que un enfoque de responsabilización con altas consecuencias - al que denominan "reformas basadas en estándares"- podría entregar al sistema educativo chileno en su intento por generar procesos de mejoramiento. Al respecto, los autores indican que el principal elemento destacable de esta estrategia es su eficacia, en términos del efecto en el mejoramiento en los resultados de aprendizaje. Argumentan a favor de la existencia de dispositivos complementarios a los estándares que proporcionarían los apoyos necesarios para que las escuelas puedan impulsar su mejoramiento. En contraposición, en el mismo libro Casassús (2010) cuestiona en su capítulo dichas políticas, debido al aumento del control del Estado que implican los estándares y la rigidez de dichas definiciones curriculares con relación al efecto que implican en la toma de decisiones de las escuelas.

En términos de la información disponible sobre los efectos de los sistemas de accountability educacional existentes en Chile, Carrasco, Perez y Núñez (2015) encuentran que los establecimientos adscritos a la SEP, luego de cuatro años de implementación, incrementan sus puntajes SIMCE en 4to básico, lo cual es significativamente mayor en la prueba de matemáticas en comparación a los establecimientos de control ( $\sin$ SEP o con ingreso tardío). Mizala y Torche (2013), consistente al estudio anterior, examinan la evolución de los resultados SIMCE en escuelas particulares subvencionadas adscritas a la SEP, y encuentran que los establecimientos incrementan progresivamente sus puntajes en promedio. Este aumento es especialmente mayor en el quintil más pobre; mientras, en el quintil más rico no se observa cambios estadísticamente significativos.

Por su parte, Valenzuela, Villarroel y Villalobos (2013), que estudian escuelas municipales y particulares subvencionadas con hasta 3 años de participación en la ley SEP, también identifican un aumento en los resultados SIMCE, pero con una evaluación menos favorable que en los estudios anteriores. En este caso, los autores identifican que el aumento se da particularmente en escuelas autónomas, así como también en escuelas con alta concentración de alumnos prioritarios, aunque en este caso el aumento es bastante más modesto que en el anterior. Con ello, se muestra que el efecto de la participación en SEP se caracteriza por la heterogeneidad.

También se han desarrollado estudios de caso y etnográficos cuyo objetivo ha sido analizar los efectos de las políticas de responsabilización por desempeño escolar al interior de los establecimientos (Acuña et al., 2014; Assaél et al., 2014; Carrasco, 2010; Falabella, 2013, 2014b, 2016). Estos estudios muestran efectos complejos y actores tensionados por cumplir las metas exigidas. La contribución de los estudios cualitativos ha sido comprender no solo los cambios en las prácticas cotidianas de las escuelas, sino también en las subjetividades de los actores. Estas investigaciones también evidencian la existencia de estrategias como la reducción y uniformidad curricular, el adiestramiento para pruebas estandarizadas y el empleo de soluciones rápidas que otorgan desempeños visibles, en lugar de cambios profundos y de largo plazo. Esta situación es confirmada en estudios de orden cuantitativo (CIDE, 2012; Elacqua et al., 2013; Manzi et al., 2014 ${ }^{11}$ ).

\footnotetext{
La encuesta CIDE (2012), por ejemplo, muestra que un 58,6\% de los docentes afirma que el SIMCE genera una mayor mecanización en los procesos de enseñanza-aprendizaje, un 67,8\% que produce una focalización en las asignaturas evaluadas por esta prueba y un 70,9\% que se le da una importancia exagerada. En una encuesta a directores, Manzi et al. (2014) identifican estrategias específicas para la preparación de dicha prueba, como la realización de ensayos (97\%) y la implementación de talleres dirigidos a todos los alumnos para reforzar los contenidos evaluados por el SIMCE (66,5\%).
} 
Una reciente investigación de Falabella y Opazo (2014), que estudia en profundidad ocho establecimientos, indica que los dispositivos de este tipo de políticas gatillan una racionalidad instrumental en los actores de las escuelas, ya que se intenta asegurar mayores logros en los resultados evaluativos en desmedro de criterios pedagógicos y éticos, lo que genera dilemas en los actores. Se evidencia, además, que los efectos de la política varían significativamente de acuerdo al grado de des/ventaja de las escuelas en su posicionamiento en el mercado escolar ${ }^{12}$, a la mediación de la política de parte del sostenedor y al ethos de la escuela y su sintonía con los supuestos y enfoques de la política nacional orientada al logro de metas estandarizadas.

Los hallazgos del estudio señalan que en las instituciones escolares con un posicionamiento aventajado en el mercado escolar y con proyectos educativos de alto rendimiento académico, la política puede pasar casi inadvertida y más bien valida y refuerza el estilo de trabajo (a pesar de la existencia de prácticas de selección y exclusión). Por otra parte, aquellos establecimientos con bajo rendimiento y situados en contextos de mayor desventaja social, así como aquellos con proyectos educativos "alternativos" no tradicionales, experimentan mayores tensiones entre las necesidades locales, el proyecto educativo y el cumplimiento de metas establecidas. Es en las escuelas urbanas marginales con bajo rendimiento donde se identifican los efectos más nocivos de esta política. Por último, en los establecimientos situados en contextos de mayor aislamiento y/o baja competencia por matrícula ${ }^{13}$ hay una disminuida percepción de presión externa por el desempeño, al tiempo que se valora el apoyo externo que se pudiera recibir desde la política.

En síntesis, existen estudios nacionales que muestran que las políticas de responsabilización por desempeño — como en el caso de escuelas adscritas a la SEP- han supuesto aumentos en los resultados en la prueba SIMCE. Sin embargo, estos hallazgos no son definitivos e implican avances diferenciados según tipo de establecimientos. Por otra parte, investigaciones de corte cualitativo dan luces sobre consecuencias complejas en las prácticas escolares, que cuestionan las expectativas iniciales de este tipo de política. De todos modos, se trata de estudios iniciales, por lo que se requiere de un mayor cúmulo de investigaciones, tanto cuantitativas como cualitativas, que examinen las interacciones entre la política nacional, los contextos socio-educativos y las prácticas escolares, y cómo estas se desarrollan a través del tiempo.

\section{DISCUSIÓN FINAL}

Estudiar las políticas de responsabilización por desempeño o con altas consecuencias en Chile es crucial para comprender cómo operan estos mecanismos de regulación en el sistema escolar. En este artículo, se examinaron, en primer lugar, los componentes y supuestos que están a la base del modelo, lo que permitió discutir sus fundamentos y levantar interrogantes sobre ciertas nociones que se han naturalizado en los discursos de esta política. Asimismo, se identificaron distintos enfoques de responsabilidad educativa, lo que evidenció que la rendición de cuentas no es un componente exclusivo de los países

\footnotetext{
Ello incluye el prestigio local de la institución, el grado de demanda de parte de las familias y los resultados académicos.

13 Como podría ser una escuela rural o una escuela particular subvencionada con un único dueño y con un "nicho" de padres estables.
} 
que han incorporado el esquema de responsabilización por desempeño, sino que, más bien, existen distintos idearios y modos de combinar las políticas en cuestión.

En segundo lugar, se realizó una revisión bibliográfica de las evidencias empíricas respecto de los efectos del modelo de responsabilización por desempeño, tanto a nivel internacional como nacional. La expectativa inicial es que la combinación de las políticas en cuestión genera incentivos adecuados para que los equipos educativos mejoren continuamente los procesos educativos. Sin embargo, aunque algunos autores identifican avances o aspectos positivos — como por ejemplo, mayor monitoreo de los aprendizajes de los niños y mayor alineación entre el currículum nacional y el trabajo en aula- existe una extensa literatura especializada que evidencia significativas tensiones e, incluso, efectos nocivos para el desarrollo de las capacidades internas de las instituciones, la profesionalización de los docentes, la calidad de las prácticas pedagógicas y las prácticas de equidad e inclusión social.

En tercer lugar, este análisis permite repensar la política y sus posibles transformaciones. Al respecto, por ejemplo, Hargreaves (2010) argumenta que se debe avanzar hacia un modelo similar al de los países nórdicos y asiáticos, que han puesto el foco prioritario en la formación y condiciones laborales de los docentes, al cual han llamado "la cuarta vía" (Hargreaves y Shirley, 2012). Sahlberg (2011), por su parte, hace referencia al caso finés, sosteniendo la relevancia de que los logros educacionales se deban justamente a la alta profesionalización y autonomía de los docentes y escuelas, junto con una educación pública extensa y una fuerte ética por la equidad y derecho sociales. Otro caso interesante de examinar es Bélgica, que ha incorporado pruebas a nivel local que incluyen la participación de docentes en la elaboración de los mismos instrumentos (Dumay et al., 2013). En la misma línea se destaca el caso de Escocia, que, aunque ha introducido políticas de planificación y evaluación estandarizada, pone el acento en la reflexión interna de los equipos escolares y su desarrollo profesional (Ozga y Grek, 2012).

Respecto de la situación en Chile, esta área de investigación es aún incipiente. Sin embargo, cobra especial relevancia el estudio de las características y los efectos de las políticas de responsabilización por desempeño o de rendición de cuentas debido a la importancia progresiva que han adquirido en las últimas décadas, más aún si se consideran los cambios introducidos producto del surgimiento de la Superintendencia de Educación, la Agencia de Calidad de la Educación y los modelos evaluativos y de ordenación asociados a ellas. Por su parte, el análisis de otros enfoques a nivel internacional resulta sugerente para analizar críticamente la política de aseguramiento de la calidad en Chile y ampliar los imaginarios políticos de posibles cambios.

\section{REFERENCIAS BIBLIOGRÁFICAS}

Acuña, F., Assaél, J., Contreras, P., \& Peralta, B. (2014). La traducción de los discursos de la política educativa en la cotidianeidad de dos escuelas municipales chilenas: La metáfora médica como vía de análisis. Psicoperspectivas. Individuo y Sociedad, 13(1), 46-55. doi:10.5027/ PSICOPERSPECTIVAS-VOL13ISSU1-FULLTEXT-363

Altrichter, H., \& Kemethofer, D. (2015). Does accountability pressure through school inspections promote school improvement? School Effectiveness and School Improvement, 26(1), 32-56.

Anderson,P.(2011). National policy and the implementation of recognition of prior learning in a Swedish municipality. Journal of Education Policy, 23(5), 515-531. doi:10.1080/02680930802209727 
Estudios Pedagógicos XLII, N²: 395-413, 2016

POLÍTICAS DE RESPONSABILIZACIÓN POR DESEMPEÑO ESCOLAR: UN DEBATE A PARTIR DE LA LITERATURA INTERNACIONAL Y EL CASO CHILENO

Apple, M. (Ed.). (2003). The state and the politics of the knowledge. New York, London: Routledge Falmer.

Assaél, J., Acuña, F., Contreras, P., \& Corbalán, F. (2014). Transformaciones en la cultura escolar en el marco de la implementación de políticas de accountability en Chile. Un estudio etnográfico en dos escuelas clasificadas en recuperación. Estudios Pedagógicos, 40(2), 7-26. Recuperado desde http://dx.doi.org/10.4067/S0718-07052014000300001

Ball, S.J. (2006). Educational reform, market concepts and ethical re-tooling. In S.J. - Ball, Education Policy and Social Class. The selected works of Stephen Ball (pp. 116-119). London, New York: Routledge.

Barber, M. (2004). The Virtue of Accountability: System Redesign, Inspection and Incentives in the Era of Informed Professionalism. Journal of Education, 185(1), 7-38.

Barber, M. (2010). How Government, Professions and Citizens Combine to Drive Successful Educational Change. In A. Hargreaves, A. Lieberman, M. Fullan, \& D. Hopkins (Eds.), Second International Handbook of Educational Change (Vol. 1, pp. 261-278). Dordrecht: Springer Science+Business Media.

Bruns, B., Filmer, D., \& Patrinos, H. (2011). Making schools work: new evidence on accountability reforms. Washington, DC: World Bank.

Carnoy, M., \& Loeb, S. (2003). Does external accountability affect student outcomes? A cross-state analysis. Education Evaluation and Policy Analysis, 24(4), 305-331. doi:10.3102/01623737024004305

Carrasco, A. (2010). A case-study of the Chilean policy agenda for disadvantaged primary schools: meeting their challenges? (Unpublished doctoral dissertation). University of Cambridge.

Carrasco, A., Falabella, A., \& Seppänen, P. (2015). Educational accountability policy schemes in Chile and Finland. In P. Seppänen, A. Carrasco, M. Kalalahti, R. Rinne, \& H. Simola (Eds.), Contrasting Dynamics in Education Politics of Extremes: school choice in Finland and Chile (pp. 53-80). Rotterdam: SENSE.

Carrasco, R., Pérez, M., \& Núñez, D. (2015). Hacia una distribución más equitativa de las oportunidades educativas: ¿Cuál es el impacto de la política de Subvención Preferencial en el desempeño académico de los alumnos más vulnerables en Chile? Pensamiento Educativo. Revista de Investigación Educacional Latinoamericana, 52 (1), 65-83.

Casassús, J. (2010). Las Reformas Basadas en Estándares: un camino equivocado. En C. Bellei, D. Contreras, \& J. Valenzuela, (Eds.), Ecos de la revolución pingüina. Avances, debates y silencios en la reforma educacional (pp. 51-83). Santiago: Universidad de Chile.

CIDE. (2012). IX Encuesta a Actores del Sistema Educativo. Recuperado desde http://www.cide.cl/ documentos/Informe_IX_Encuesta_CIDE_2012.pdf

Darling-Hammond, L. (1994) National standards and assessments: Will they improve? American Journal of Education, 102(4), 478-510.

Darling-Hammond, L. (2007). Race, Inequality and Educational Accountability: The Irony of "No Child Left Behind". Race, Ethnicity and Education, 10(3), 245-260.

Darling-Hammond, L. (2010). Teaching and Educational Transformation. En A. Hargreaves, A. Lieberman, M. Fullan, \& D. Hopkins (Eds.), Second International Handbook of Educational Change (pp. 505-520). Dordrecht, Heidelberg, London, New York: Springer Science+Business Media.

Darling-Hammond, L., Wilhoit, G., \& Pittenger, L. (2014). Accountability for college and career readiness: Developing a new paradigm. Education Policy Analysis Archives, vol. 22 (86). doi:http://dx.doi.org/10.14507/epaa.v22n86.2014

Dee, T., Jacob, B., Hoxby, C., \& Ladd, H. (2010). The Impact of No Child Left Behind on Students, Teachers, and Schools. Brookings Papers on Economic Activity, Fall 2010, 149- 207.

di Gropello, E. (2004). Education Decentralization and Accountability Relations in Latin 
America (World Bank Policy Research working paper 3453). Recuperado desde http:// www.oei.es/reformaseducativas/descentralizacion_educacion_rendicion_cuentasAL_ digropello.pdf

Dumay, X., Cattonar, B., Maroy, C., \& Mangez, C. (2013). The local institutionalization of accountability in education: Network and bureaucratic modes of implementation. International Journal of Sociology of Education, 2(2), 99-141. doi:10.4471/ rise. 2013.26

Dupriez, V., \& Dumay, X. (2011). Les quasi-marches scolaires : au benefice de qui? Revue française de pédagogie: recherches en éducation, 176, 83-100.

Ehren, M., \& Hatch, T. (2015). Responses of schools to accountability systems using multiple measures: the case of New York City elementary schools. Educational Assessment, Evaluation and Accountability, 25(4), 341-373.

Elacqua, G., Martínez, M., Santos, H., \& Urbina, D. (2013). Escuelas bajo amenaza: Efectos de corto plazo de las presiones de accountability de la Ley SEP en las políticas y prácticas docentes (Documento de trabajo 16). Recuperado desde http://politicaspublicas.udp. cl/media/publicaciones/archivos/361/ESCUELAS_BAJO_AMENAZA_EFECTOS_ DE_CORTO_PLAZO_DE_LAS_PRESIONES_DE_ACCOUNTABILITY_DE_LA_ LEY_SEP_EN_LAS_POLITICAS_Y_PRACTICAS_DOCENTES.pdf

Espínola, V., \& Claro, J. (2010). El sistema nacional de aseguramiento de la calidad: una reforma basada en estándares. En C. Bellei, D. Contreras, \& J. Valenzuela (Eds.), Ecos de la revolución pingüina. Avances, debates y silencios en la reforma educacional (pp. 51-83). Santiago: Universidad de Chile.

Eyzaguirre, B., \& Fontaine, L. (2008). Las escuelas que tenemos. Santiago: CEP.

Falabella, A. (2007). Des-centralización en educación. Relaciones de Amor Odio con el Estado. Revista Docencia, 31, 12-21.

Falabella, A. (2013). Accountability policy effects within school markets. A study in three Chilean municipalities (Unpublished doctoral dissertation). Institute of Education, University of London.

Falabella, A. (2014a). Do national test scores and quality labels trigger school selfassessment and accountability? A critical analysis in the Chilean context. British Journal of Sociology of Education. Recuperado desde http://dx.doi.org/10.1080/014 25692.2014.976698

Falabella, A. (2014b). Do national test scores and quality labels trigger school selfassessment and accountability? A critical analysis in the Chilean context. British Journal of Sociology of Education. Recuperado desde http://dx.doi.org/10.1080/014 25692.2014.976698

Falabella, A. (2016). ¿Qué aseguran las políticas de aseguramiento de la calidad? Un estudio de casos en distintos contextos escolares. Estudios Pedagógicos, 42(1).

Falabella, A., \& Opazo, C. (2014). Sistema de Aseguramiento de la Calidad y procesos de mejora: Una mirada desde la gestión educativa. Informe ejecutivo. Recuperado desde http://biblioteca.uahurtado.cl/ujah/reduc/pdf/pdf/txt1107.pdf

Figlio, D., \& Loeb, S. (2011). School Accountability. In E. Hanushek, S. Machin, \& L. Woessmann (Eds.), Handbook of Economics of Education (Vol. 3, pp. 383.423). San Diego: North Holland. doi: 10.1016/S0169-7218(11)03008-5

Finnigan, K., \& Gross, B. (2007). Do Accountability Policy Sanctions Influence Teacher Motivation? Lessons from Chicago's Low-Performing Schools. American Educational Research Journal, 44 (3), 594-629. doi:10.3102/0002831207306767

Firestone, W., Schorr, R., \& Monfils, L. (Eds.). (2004). The ambiguity of teaching to the test: standards, assessments and educational reform. Mahwah: Lawrence Erlbaum.

Gewirtz, S. (2002). The managerial school: postwelfarism and social justice in education. 
Estudios Pedagógicos XLII, N 2: 395-413, 2016

POLÍTICAS DE RESPONSABILIZACIÓN POR DESEMPEÑO ESCOLAR: UN DEBATE A PARTIR DE LA LITERATURA INTERNACIONAL Y EL CASO CHILENO

London: Routledge.

Hamilton, L., Stecher, B., Marsh, J., Sloan McCombs, J., Robyn, A., Lin Russell, J., Naftel, S., \& Barney, H. (2007). Standards-based accountability under No Child Left Behind. Experiences of teachers and administrators in three states. Santa Monica, CA: RAND Corporation.

Hamilton, L., Stecher, B., \& Yuan, K. (2012). Standards-Based Accountability in the United States: Lessons Learned and Future Directions. Educational Inquiry, 3(2), 149-170.

Hanushek, E., \& Woessmann, L. (2010). How Much Do Educational Outcomes Matter in OECD Countries? Paper presented at the 52th Panel Meeting of Economic Policy, Rome.

Hargreaves, A. (2010). Change from Without: Lessons from Other Countries, Systems, and Sectors. In A. Hargreaves, A. Lieberman, M. Fullan, \& D. Hopkins (Eds.), Second International Handbook of Educational Change (Vol. 1, pp. 105-117). Dordrecht, Heidelberg, London, New York: Springer Science+Business Media.

Hargreaves, A., \& Fink, D. (2006). Estrategias de cambio y mejora en educación caracterizadas por su relevancia, difusión y continuidad en el tiempo. Revista de Educación, 339, 43-58.

Hargreaves, A., \& Shirley, D. (2012). La cuarta vía. El prometedor futuro del cambio educativo. Barcelona: Octaedro.

Jacob, B. (2009). Test- based accountability and student achievement: an investigation of differential performance on Naep and State assesments (CLOSUP Working Paper Series Number 17).

Jofré, G. (1988). El Sistema de subvenciones en educación: La experiencia chilena. Revista Estudios Públicos, 32, 193-237. Recuperado el 15 de Septiembre de 2014 desde http:// www.cepchile.cl/dms/archivo_1599_1154/rev32_jofre.pdf

Koretz, D. (2005). Alignment, high stakes, and the inflation of test scores. Boston: Harvard School of Education.

Lazzarato, M. (2012). Neoliberalism in action. Inequality, insecurity and the reconstruction of the social. Theory, Culture and Society, 26(6), 109-133.

Lipman, P. (2004). High stakes education: inequality, globalization, and urban school reform. New York: Routledge.

Looney, J. (2009). Assessment and innovation in education (OECD Education Working Paper No. 24).

MacBeath, J. (2009). Self evaluation for school improvement. En A. Hargreaves, A. Lieberman, M. Fullan, \& D. Hopkins, D. (Eds.), Second International Handbook of Educational Change (pp. 901-912). Netherlands: Springer.

MacBeath, J. (2011). Liderar el aprendizaje dentro y fuera de la escuela. Santiago: Fundación Chile.

MacDermott, K. (2007). "Expanding the Moral Community" or "Blaming the Victim"? The Politics of State Education Accountability Policy. American Educational Research Journal, 44(1), 77 111. doi:10.3102/OO0002831206299010

Manno, B. (2005). Chartering and the Idea of Accountability Consequences: Adding Performance Value to Schooling. Conferencia presentada en el Seminario Internacional Accountability Educacional: posibilidades y desafíos para América Latina a partir de la experiencia internacional, Santiago. Recuperado desde http://m.preal.org/detalle.asp?det=496

Manzi, J., Bogolasky, F., Gutiérrez, G., Grau, V., \& Volante, P. (2014). ¿Cuánto conocen, usan y valoran el SIMCE los directores escolares? Informe Proyecto FONIDE- MINEDUC, Santiago.

Maroy, C. (2009). Convergences and hybridization of educational policies around 'post-bureaucratic' models of regulation. Compare: A Journal of Comparative and International Education, 39(1), 71-84. doi:10.1080/03057920801903472

Mintrop, H., \& Trujillo, T. (2007). The practical relevance of accountability systems for school improvement: A descriptive analysis of California Schools. Educational Evaluation and Policy 
Analysis, 29(2), 319-352. doi:10.3102/0162373707309219

Mizala, A., \& Torche, F. (2013). ¿Logra la subvención escolar preferencial igualar los resultados educativos? Espacio Público, documento de referencia $n^{\circ}$ 9. Recuperado desde http://www. espaciopublico.cl/publicaciones/detalle.tpl?id=19

Mourshed, M., Chijioke, C., \& Barber, M. (2010). How the world's most improved school systems keep getting better. NY: McKinsey \& Company.

National Research Council. (2011). Incentives and Test-Based Accountability in Education. Committee on Incentives and Test-Based Accountability in Public Education, M. Hout and S.W. Elliott (Eds.). Board on Testing and Assessment, Division of Behavioral and Social Sciences and Education. Washington, DC: The National Academies Press.

Newman, J. (2005). Remaking governance. Peoples, politics and the public sphere. Bristol: The Policy Press, University of Bristol.

Ozga, J. (2011). Governing narratives: 'local' meanings and globalising education policy. Education Inquiry, 2(2), 305-318. Recuperado desde http://dx.doi.org/10.3402/edui.v2i2.21982

Ozga, J., \& Grek, S. (2012). Governing through learning. School self-evaluation as a knowledgebased regulatory tool. Recherches Sociologiques et Anthropologiques, 43(2), 35-52.

Power, S., \& Frandji, D. (2010). Education markets, the new politics of recognition and the increasing fatalism towards inequity. Journal of Education Policy, 25(3), 385-396.

Prieto, A. (1983). La modernización educacional. Santiago: Ediciones Universidad Católica de Chile. Puryear, J. (2006). La accountability en la educación: ¿Qué hemos aprendido? In J. Corvalán, \& R. McMeekin (Eds.), Accountability educacional: posibilidades y desafios para América Latina a partir de la experiencia internacional (pp. 125-133). Santiago: CIDE- PREAL. Recuperado desde http://biblioteca.uahurtado.cl/UJAH/Reduc/pdf/pdf/mfn268.pdf

Ravitch, D. (2010). The Death and Life of the Great American School System: How Testing and Choice Are Undermining Education. New York: Basic Books.

Rose, N., \& Miller, P. (1992). Political Power beyond the State: Problematics of Government. The British Journal of Sociology, 43(2), 173-205.

Sahlberg, P. (2011). Finnish Lessons: What can the world learn from educational change in Finland? Columbia: Teachers College Press.

Thrupp, M., \& Lupton, R. (2006). Taking school context more seriously: The social justice challenge. British Journal of Educational Studies, 54(3), 308-328. doi:10.1111/j.1467-8527.2006.00348.x.

Valli, L., \& Buese, D. (2007). The Changing Roles of Teachers in an Era of High-Stakes Accountability. American Educational Research Journal, 44(3), 519-558. doi:10.3102/0002831207306859

Valenzuela, J., Villarroel, G., \& Villalobos, C. (2013). Ley de Subvención Escolar Preferencial (SEP). Algunos resultados preliminares de su implementación. Revista Pensamiento Educativo, 50(2), 113-131.

Winkler, D. (2006). Fortalecer accountability (rendición de cuentas) en la educación pública. En J. Corvalán, \& R. McMeekin (Eds.), Accountability educacional: posibilidades y desafíos para América Latina a partir de la experiencia internacional (pp. 99-123). Santiago: CIDE- PREAL. Recuperado desde http://biblioteca.uahurtado.cl/UJAH/Reduc/pdf/pdf/mfn268.pdf

Woods, P., \& Jeffrey, B. (2002). The reconstruction of primary teachers' identities. British Journal of Sociology of Education, 22(1), 89-106.

Yaekyoung, L. (2008). Is Test- Driven Testing accountability Effective? Synthetizing the evidence from Cross State Causal Comparative and correlational studies. Review of Educational Research, 78(39), 608- 644. doi:10.3102/0034654308324427

Youdell, D. (2004). Engineering school markets, constituting schools and subjectivating students: the bureaucratic, institutional and classroom dimensions of educational triage. Journal of Education Policy, 19(4) 407-431. doi:10.1080/0268093042000227474 
